\title{
Attributing human-likeness to an avatar: the role of time and space in the perception of biological motion
}

\author{
Davide Ghiglino $^{1-2[0000-0002-7825-732 X]}$, Davide De Tommaso ${ }^{10000-0003-2132-5261]}$ and \\ Agnieszka Wykowska ${ }^{10000-0003-3323-7357]}$ \\ ${ }^{1}$ Istituto Italiano di Tecnologia, Via Morego, 30, 16163, Genova, Italy \\ 2 DIBRIS, Università degli Studi di Genova, Via Opera Pia 13, 16145, Genova, Italy
}

Manuscript accepted for publication in proceedings of the International Conference for Social Robotics (ICSR), 2018, Qingdao, China. Proceedings will be published in the Springer LNCS/LNAI series 


\begin{abstract}
Despite well-developed cognitive control mechanisms in most adult healthy humans, attention can still be captured by irrelevant distracting stimuli occurring in the environment. However, when it comes to artificial agents, such as humanoid robots, one might assume that its attention is "programmed" to follow a task, thus, being distracted by attention-capturing stimuli would not be expected. We were interested in whether a behavior that reflects attentional capture in a humanoid robot would increase its perception as human-like. We implemented human behaviors in a virtual version of iCub robot. Twenty participants' head movements were recorded, through an inertial sensor, during a solitaire card game, while a series of distracting videos were presented on a screen in their peripheral field of view. Eight participants were selected, and their behavioral reactions (i.e. inertial sensor coordinates, etc.) were extracted and implemented in the simulator. In Experiment 2, twenty-four new participants were asked to rate the human-likeness of the avatar movements. We examined whether movement parameters (i.e. angle amplitude, overall time spent on a distractor) influenced participants' ratings of human-likeness, and if there was any correlation with sociodemographic factors (i.e. gender, age). Results showed a gender effect on human-likeness ratings. Thus, we computed a GLM analysis including gender as a covariate. A main effect of the time of movement was found. We conclude that humans rely more on temporal then on spatial information when evaluating properties (specifically, human-likeness) of biological motion of humanoidshaped avatars.
\end{abstract}

Keywords: Human-likeness of robot behavior, Biological Motion, Humanoid robots.

\title{
1 Introduction
}

In designing artificial agents that are to appear human-like in order to increase perceived naturalness and facilitate social attunement, many researchers address the issue of creating human-like behavior. Several characteristics have been identified, and one crucial characteristic is variability [1]: behavioral observations demonstrate that humans never display exactly the same behavior twice. For example, several studies demonstrated that subjects tend to adopt unique patterns of kinematic strategies to attend the very same target [2-5]. The recent advent of complex humanoid systems, allow researchers to implement fragmentized human behaviors in artificial agents, in order to study in more detail on what type of information humans rely most when evaluating biological motion. Furthermore, a deeper understanding of human perception of synthetic motion will facilitate, in the future, human-robot interaction [6]. This stems in part from the fact that humans, when interacting with other mammals [7], easily understand goals, motivation and beliefs behind human-like behaviors [8], also relying on motion clarity. It is not clear whether artificial motion patterns of a robot would be as easily understood and predicted. Therefore, it is of high importance to examine what parameters of robot behavior make it well-understood by the human users. Evidence from literature pointed out that motion cues might influence social attunement with artificial agents, enhancing even empathetic and mentalizing processes [9-11]. Starting 
with observing and recording human motion, several techniques can be used to transfer movement parameters to artificial agents [12-14]. However, given the huge variability of humans' motion, it is still unclear which components of observed behaviors affect most the perception of human-likeness. The projection of human motion in simulation environment might be a suitable method to systematically study these factors.

\subsection{Aim of study}

The goal of the present study was to investigate how human participants perceive biological movement displayed by an artificial agent, in terms of human-likeness. We selected an attention-capture scenario, because attention capture seems to be a very human-like phenomenon. Humans (and several other animal species) have developed mechanisms to attend relevant events in the environment. The "decision" of the brain to attend to a given event in the environment is made through a combination of bottomup characteristics of the stimulus (e.g., the salience of the stimulus) and internal topdown factors of the agent (e.g., bias towards emotional stimuli, or towards a particular sound of, for example, one's own child's voice). However, in many cases, the brain attends stimuli that "capture" attention through their salience, although this disrupts a given task. Think, for example, of driving. The driver should be focused on the road ahead of him/her, and on keeping the car in the assumed lane. However, if there is a very loud distracting sound or bright light flashing in the peripheral vision, the driver might be distracted by this event, and in consequence, lose focus on the task, potentially causing an accident. Therefore, although evolutionarily adaptive, the attentional capture phenomenon can be disruptive for a task. In this context, one might think that artificial intelligence should be better adapted to successful completion of a given task, and not allow being distracted by peripheral events that might result in sub-optimal performance in a task. We reasoned, that "being distracted" - especially with variable ways of reacting to the distracting stimuli might be perceived as an essentially human-like feature. We therefore set out to test if equipping a humanoid robot with behaviors reflecting attentional capture would make it perceived as human-like, and which particular aspects of the behavior would be crucial for attributions of human-likeness. To this aim, we recorded human head- and eye movements during an attentional capture paradigm. The recorded behaviors were filtered, and eight different movement profiles were implemented on an $\mathrm{iCub}$ [15] simulator. Then, a group of participants was asked to rate the human-likeness of the movements of the simulator.

\section{Materials and Methods}

\subsection{Attentional capture with humans}

Selection of Distracting Stimuli. Sixty HD quality video were selected from YouTube according to the following criteria: (1) presence of a single salient sound in the whole sequence (i.e. a phone ring, a woman laugh, a door slam, etc.); (2) absence of inappropriate contents (i.e. politic, racism, sexism, etc.); (3) more than $100 \mathrm{M}$ views. Selected videos were edited using Apple Final Cut Pro [16], in order to make all of them last for 
the same amount of time (twenty seconds). Fifty-five anonymous Italian participants were asked to rate the emotional content of the videos through an online platform (soscisurvey.de, [17]), using a ten-point Likert scale $(0=$ not emotional at all; $10=$ strongly emotional). One subgroup $(\mathrm{n}=26)$ was asked to rate only the audio tracks of the videos. The other subgroup $(n=30)$ was asked to rate both the audio and the visual component of the videos. After collecting the data, ratings of the two groups were compared. Four of the sixty initial stimuli were excluded because of the inconsistency between ratings of the two groups. The remaining fifty-six videos were categorized into "Affective" and "Non-Affective" stimuli, using the median score of the raters as cutoff value between the two categories. Eighteen videos were then extracted, according to the following criteria: the nine with the lowest score ("Non-Affective" videos) and the nine with the highest score ("Affective" videos). By using Apple Final Cut Pro, audio tracks of the final eighteen videos were manipulated, in order to increase the salience of one single sound per video (i.e. the phone ring, the woman laugh, the door slam). Furthermore, we edited the videos in order to ensure that the physical properties of the sounds (i.e. volume and sampling rate, $44.1 \mathrm{kHz}$ ) were consistent. For each video, the volume of the single salient sound was increased, while all the other sounds were reduced. The final pool of videos was implemented in an attentional capture paradigm as distracting stimuli.

\subsection{Experiment 1.}

Participants. Twenty-two healthy young adults (9 females; 19-34 years of age) were recruited. All participants were native Italian language speakers with no history of psychiatric or neurological diagnosis, substance abuse or psychoactive medication. All participants had normal or corrected-to-normal vision and reported no history of hearing impairment. Experimental protocols followed the ethical standards laid down in the Declaration of Helsinki and the local Ethics Committee (Comitato Etico Regione Liguria) approved procedures. Each participant provided written informed consent to participation in the experiment. Participants were not informed regarding the purpose of the study before the experiment, but were debriefed upon completion.

Experimental design. Participants were seated in a sound attenuated experimental booth with dimmed light, in front of a notebook screen (HP Stream 14-ax011nl, 1366 x 768) (Fig. 1). 


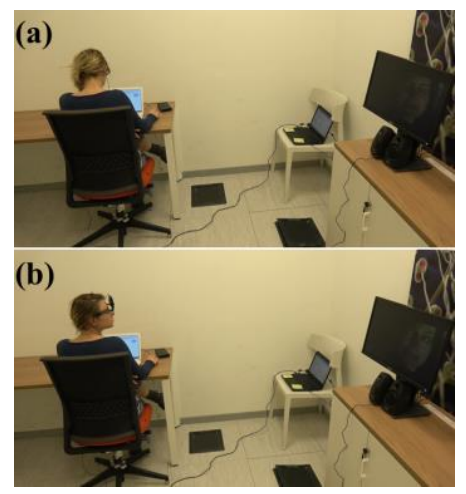

Fig. 1. Experimental setup: (a) participant is engaged in a solitaire game on the laptop; (b) participant reacts to a distracting stimulus.

Participants were instructed to perform a solitaire card game (spider one-suit) on the notebook, and to pay attention to the game. While participants were engaged in the card game, distracting stimuli were presented in the far periphery of their field of view $\left(100^{\circ}\right.$ on the right, $227 \mathrm{~cm}$ of distance), on a second computer screen (DELL S2716DG, 2560 x 1440 pixels), interleaved by soft ambient sounds lasting for five second each (i.e. gentle rain). We exposed all participants to the same randomized sequence of distractors. The audio tracks of the distracting stimuli were played through loudspeakers (Logitech LGT-Z130), located under the second screen. The experiment was programmed and run on OpenSesame [18]. Participants' eye movements were recorded throughout the entire duration of the experiment with a mobile eye-tracking device [19]. Head movements were recorded using an inertial sensor (Bosch Sensortec BNO055 Intelligent 9-Axis Absolute Orientation Sensor, [20]) mounted on the eye-tracker and integrated in the OpenSesame experiment. We implemented a periodic task, running at $50 \mathrm{~Hz}$, that requests every $20 \mathrm{~ms}$ the Euler angles to the inertial sensor. The absolute values of these angles, together with the sample timestamp (Timestamp, Yaw, Pitch and Roll) were saved in a .csv file, one for each distractor stimulus. Specifically, the periodic task was synchronized with the video stimuli, so that the duration of each inertial measure was aligned with the duration of the video. For each experiment, we collected 18 sessions for each participant, in total 360 .csv files.

Data Analyses. Participants' data were extracted from the eye-tracker and from the inertial sensor through Tobii Pro Lab and OpenSesame, respectively. Two participants were excluded due to noisy data. Participants' reactions to distracting stimuli were defined as head rotations of at least $30^{\circ}$ the on the yaw axis (horizontal plane) of the inertial sensor. Reactions of participants were treated and analyzed as a count variable. For each subject, three final parameters were extracted: (1) total amount of distractions during the whole experiment, (2) total amount of distractions occurring during "Affective" stimuli, and (3) total amount of distractions occurring during "Non-Affective" stimuli. A Wilcoxon matched pairs test was used to verify a potential difference between "Affective" and "Non-Affective" conditions. Furthermore, in order to explore gender differences in distractibility among participants, a Fisher Exact Test was used 
to compare males and females, separately for "Affective" and "Non-Affective" conditions. In order to apply the Fisher Exact Test, the number of reactions was converted into a relative percentage estimated for each single subject. Finally, a binomial test $(\mathrm{n}=20, \mathrm{p}=50 \%, 1-\alpha=.95)$ was used to identify the most distracting stimuli of our pool. Two sounds (a gunshot and a woman's orgasm) survived the .95 threshold, meaning that at least $70 \%$ of our sample reacted to that sound).

\subsection{Implementation of humans' behaviors in an iCub simulator}

Selection of behaviors. During the attentional capture paradigm, fifteen participants reacted to the sound "gun shot" and fourteen participants reacted to the sound "woman orgasm". Thus, we took into consideration the resulting twenty-nine reactions. For each reaction, we extracted two main parameters: (1) amplitude $\left(^{\circ}\right)$ of the movement; (2) time (s) spent on the distractor. The first parameter represented the angle of rotation of the head toward the distracting screen, and was calculated as the difference between the average position assumed by the head of the participant during the whole video and the maximum distance reached on the horizontal plane (yaw axis of the inertial sensor) during the same temporal window. The time spent on the distractor was estimated as the time spent by the subject on a point of the horizontal plane exceeding two standard deviations from the average position of the head. Setting this high threshold allowed us to extract thirteen reactions from the initial pool. Then, the median value of the amplitude $\left(\mathrm{Mdn}=51,108^{\circ}\right)$ and the median value of the time spent on distractor $(\mathrm{Mdn}=1,664$ s) were calculated and used as cutoff to classify the reactions. Specifically, reactions were divided in four categories, accordingly to the combination of the amplitude of the movement and the time spent on the distractor, namely:

(1) Amplitude and time above the median; (2) Amplitude above the median and time below the median; (3) Amplitude and time below the median; (4) Amplitude below the median and time below the median.

For each condition, the two most representative reactions were extracted (one for the "gun shot" and one for the "woman orgasm"). Eight reactions from eight different participants were selected as the final pool.

Reproduction of the head movements on iCub simulator. The iCub simulator (Fig. 2) has been designed to reproduce the physics and the dynamics of the robot [21].

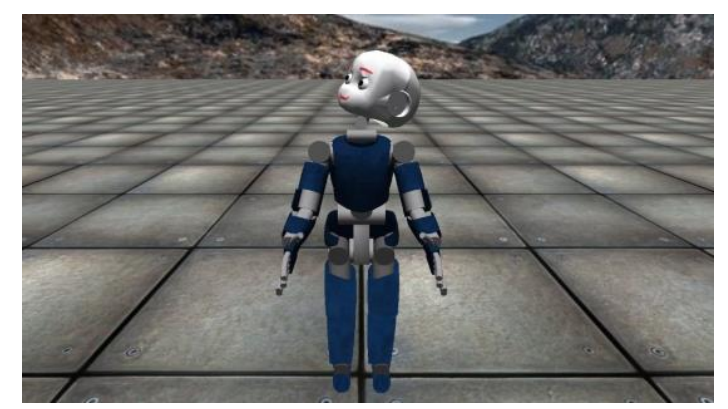

Fig. 2. Example of the iCub simulator behaviour. 
It has been implemented collecting data directly from the robot design specifications in order to achieve a replication as accurate as possible. Moreover, the software architecture is the same used to control the physical robot. Specifically, we decided to use the Direct Position Control algorithm [22] for sending the joint positions to the iCub head. According to the specifications in [23], the head joints are the ones with indexes 0,1 and 2, respectively the neck Pitch, Roll and Yaw. At first, we needed to normalize the Euler angles recorded with the inertial sensor to get relative angles with respect to the initial head pose at the onset of the stimulus. In such a way, we transferred on the robot the relative rotation due to the distractor, assuming that the head had always the same starting pose. The experiment was designed to guarantee, with good approximation, this assumption. In fact, the participants were always looking straight at the screen whenever a video stimulus occurred. We excluded all the other recordings not satisfying this condition. This preprocessing of the data was enough to reproduce on the iCub simulator the head movements using the Direct Position Control algorithm. This control technique is used whenever joint positions are sent at a high frequency, because no trajectory generation in between is needed.

\section{$2.4 \quad$ Experiment 2}

Participants. Twenty-four participants (13 females; 26-60 years of age) completed an online survey evaluating the human-likeness of iCub simulator. Data collection was conducted in accordance with the ethical standards laid down in the Code of Ethics of the World Medical Association (Declaration of Helsinki), procedures were approved by the regional ethical committee (Comitato Etico Regione Liguria).

Experimental design. Eight videos of six seconds each were recorded from the simulator. Videos were then uploaded on an online platform (soscisurvey.de) and associated with the following question: "In a scale from 1 (extremely mechanistic) to 10 (extremely human-like), how would you rate iCub behaviors in terms of human-likeness?". Each video and the associated question was presented ten times during the survey, mixed with the other items in a random order. Participants rated the human-likeness of the simulations, relying only on motion information. They were not informed that the behaviors were all based on previous recordings of humans' motions, but they were debriefed after the survey. In order to investigate whether the ratings were influenced by subjective factors, participants were also asked to complete the Empathy Quotient (EQ) questionnaire [24] after the survey.

Data analyses. A two-sample T-Test was used to assess gender differences in our sample's ratings. Pearson's correlations were applied in order to evaluate possible correlations between participants' global ratings and subjective measures (i.e. EQ).

In order to explore how the components of biological motion (amplitude of the movement and time spent on the distractor) affect ratings of human-likeness, statistical analyses were applied. Amplitude of the movement and time spent on the distractor were entered as two-level within-categorical predictors in the context of the General Linear Model (GLM). Gender of our participants was included in the model as nuisance covariate. Post hoc effects were estimated by calculating Bonferroni test. 


\section{$3 \quad$ Results}

\subsection{Experiment 1. Attentional Capture with humans}

Statistical analyses performed on the average number of reactions across participants revealed a significant difference between Non-Affective and Affective stimuli.

Specifically, Wilcoxon Matched Pairs Test detected a significant difference $(\mathrm{N}=20$, $\mathrm{T}=3.5, \mathrm{Z}=3.789, \mathrm{p}<.001$ ) between average number of reactions occurred during NonAffective stimuli ( $\mathrm{M}=2.30, \mathrm{SD}=2.00)$ and Affective stimuli ( $\mathrm{M}=5.60, \mathrm{SD}=2.46)$.

For both Non-Affective and Affective conditions, Fisher Exact Test revealed no significant effect of the gender ( $p>.05$ ) on the distractibility during the experiment (Fig. 3).

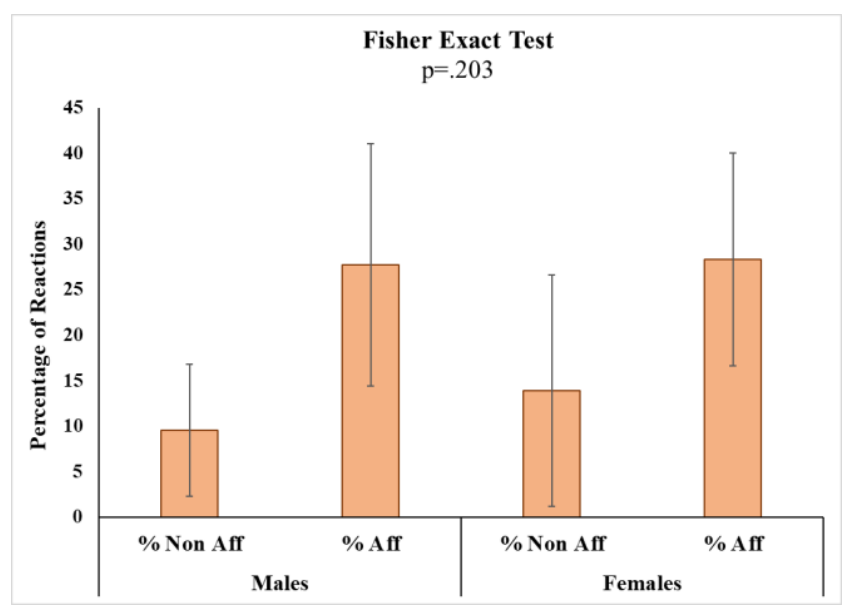

Fig. 3. Differences between males and females on the percentage of reaction displayed during Non-Affective (Non-Aff) and Affective (Aff) stimuli; vertical bars denote standard deviation of the data.

\subsection{Experiment 2. Human-likeness survey}

The two-sample T-Test revealed a significant difference $(\mathrm{T}(23)=2.425, \mathrm{p}<.05)$ of gender on the human-likeness ratings, pointing out that females $(\mathrm{M}=6.39, \mathrm{SD}=1.38)$ usually rate higher human-likeness than males $(M=4.90, S D=1.64)$. No correlation was found between ratings and subjects' Empathy Quotient scores.

The analyses modeled in the General Linear Model revealed no significant interaction between the amplitude of the movement and time spent on the distractor $(F(1,22)=$ $0.48, \mathrm{p}=.50)$. Furthermore, no main effect was found for the amplitude $(\mathrm{F}(1,22)=$ $0.18, \mathrm{p}=.68)$, although results showed significant main effect of the time spent on the distractor $(F(1,22)=9.18, p<.01)($ Fig. 4) surviving Bonferroni correction $(p<.01)$. 
Specifically, results suggest that longer time spent on the distractor might determine higher human-likeness ratings.

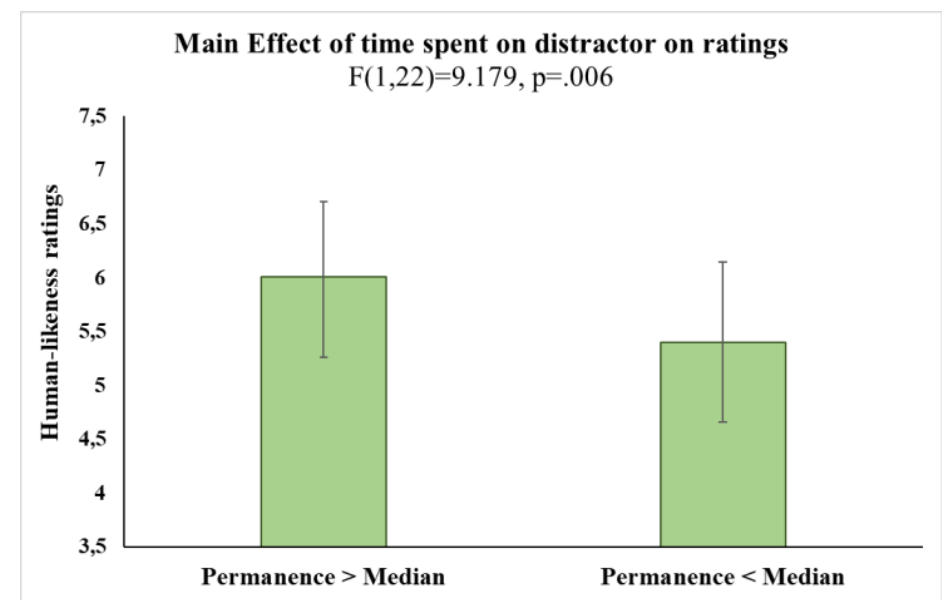

Fig. 4. Main effect of the "Time spent on distractor" on human-likeness ratings; vertical bars denote confidence intervals; Cousineau procedure was applied for correcting bars for withinparticipants comparisons.

\section{General discussion}

The aim of our study was to examine parameters of biological motion implemented on a humanoid robot avatar that determine perceived human-likeness of the motion.

In Experiment 1 we focused on the recording of human behaviors. We recorded participants' head and eye movements during an attentional paradigm. Before implementing the recorded data on an iCub simulator, a preliminary check of the data was required. Thus, we investigated whether differences between participants (males vs. females) or between conditions (Non-Affective vs. Affective) affected our results. No difference was found between males and females, suggesting that we could use all participants' recordings, regardless their gender, for subsequent implementation. At the same time, we found a difference between our experimental conditions. Specifically, results showed that Affective stimuli (i.e. a laugh, a cry, a scream, etc.) elicited more frequent reactions compared to Non-Affective ones (i.e. a phone ring, a metal drop, a door closing, etc.). We combined this result within the binomial test, in order to extract the most representative behaviors recorded during the attentional capture paradigm.

Then, eight behaviors of different participants were selected, extracted and, subsequently, implemented on an iCub simulator. An independent sample of participants was asked to rate the human likeness of the robot in the simulator, relying only on motion information. Our results showed that females' ratings of human-likeness were generally higher than males' ratings. This might suggest that females might be more prone to attribute human likeness than males to a robot simulator, regardless the physical properties of the movement displayed. In line with previous research [25] we also confirmed 
that humans, when asked to judge biological motion, rely more on temporal, than on spatial information. Interestingly, although all movement were copied from human behaviors, the average rating of participants was around 5.48 on a scale from 1 to 10 . This might suggest that regardless the naturalness of the movement, humans are still biased by additional visual information (the robot shape) when evaluating biological motion. Furthermore, a large variability was detected between participants' ratings. Despite the lack of correlation between the Empathy Quotient and the ratings, we hypothesize the existence of personality traits might influence participants' ratings. Further studies should investigate which factors might explain this variability.

\section{Conclusion}

Our results showed that temporal features of a movement are crucial in perceived human-likeness of a movement exhibited by an avatar of a humanoid robot. Thus, particular attention shall be paid on temporal trajectory when using avatars (or robots) to reproduce humans' behavior. Furthermore, large variability detected in participants' ratings of human-likeness and the gender difference suggest the necessity of investigating in more detail individual differences, especially when exploring attribution of human-likeness to an artificial agent.

\section{Acknowledgements}

This project has received funding from the European Research Council (ERC) under the European Union's Horizon 2020 research and innovation programme (grant awarded to A. Wykowska, titled "InStance: Intentional Stance for Social Attunement. Grant agreement No: 715058)

\section{References}

1. Gielniak, M. J., Liu, C. K., \& Thomaz, A. L. (2013). Generating human-like motion for robots. The International Journal of Robotics Research, 32(11), 1275-1301.

2. Freedman, E. G., \& Sparks, D. L. (2000). Coordination of the eyes and head: movement kinematics. Experimental brain research, 131(1), 22-32.

3. Stergiou, N., \& Decker, L. M. (2011). Human movement variability, nonlinear dynamics, and pathology: is there a connection?. Human movement science, 30(5), 869-888.

4. Desmurget, M., Rossetti, Y., Prablanc, C., Jeannerod, M., \& Stelmach, G. E. (1995). Representation of hand position prior to movement and motor variability. Canadian journal of physiology and pharmacology, 73(2), 262-272.

5. Sorostinean, M., Ferland, F., \& Tapus, A. (2014, October). Motion-oriented attention for a social gaze robot behavior. In International Conference on Social Robotics (pp. 310-319). Springer, Cham.

6. Wykowska, A., Chellali, R., Al-Amin, M. Md., Müller, H.J. (2014). Im-plications of Robot Actions for Human Perception. How Do We Repre-sent Actions of the Observed Robots? International Journal of Social Robotics, 6 (3), 357-366. 
7. Blakemore, S. J., \& Decety, J. (2001). From the perception of action to the understanding of intention. Nature Reviews Neuroscience, 2(8), 561.

8. Miller, L. E., \& Saygin, A. P. (2013). Individual differences in the perception of biological motion: links to social cognition and motor imagery. Cognition, 128(2), 140-148.

9. Heider, F., \& Simmel, M. (1944). An experimental study of apparent behavior. The American journal of psychology, 57(2), 243-259.

10. Frith, C. D., \& Frith, U. (1999). Interacting minds--a biological basis. Science, 286(5445), 1692-1695.

11. Pollard, N. S., Hodgins, J. K., Riley, M. J., \& Atkeson, C. G. (2002). Adapting human motion for the control of a humanoid robot. In Robotics and Automation, 2002. Proceedings. ICRA'02. IEEE International Conference on (Vol. 2, pp. 1390-1397). IEEE.

12. Lee, J., \& Lee, K. H. (2006). Precomputing avatar behavior from human motion data. Graphical Models, 68(2), 158-174.

13. Aggarwal, J. K., \& Cai, Q. (1999). Human motion analysis: A review. Computer vision and image understanding, 73(3), 428-440.

14. Metta, G., Sandini, G., Vernon, D., Natale, L., \& Nori, F. (2008, August). The iCub humanoid robot: an open platform for research in embodied cognition. In Proceedings of the 8th workshop on performance metrics for intelligent systems (pp. 50-56). ACM.

15. Apple Final Cut Pro $X$ license and download [electronic resource].

16. Leiner, D. J. (2018). SoSci Survey (Version 2.5.00-i1142) [Computer software]. Available at http://www.soscisurvey.com

17. Mathôt, S., Schreij, D., \& Theeuwes, J. (2012). OpenSesame: An open-source, graphical experiment builder for the social sciences. Behavior research methods, 44(2), 314-324.

18. TobiiAB, Stockholm (2015) 'Tobii Pro Glasses 2 Product Description'.

19. Sensortec, B. (2014). Intelligent 9-axis absolute orientation sensor. BNO055 datasheet, November.

20. http://wiki.icub.org/wiki/Simulator_README

21. http://wiki.icub.org/images/c/cf/ICub_Control_Modes_1_1.pdf

22. http://wiki.icub.org/wiki/ICub_joints\#Head_2.0

23. Baron-Cohen, S., \& Wheelwright, S. (2004). The empathy quotient: an investigation of adults with Asperger syndrome or high functioning autism, and normal sex differences. Journal of autism and developmental disorders, 34(2), 163-175.

24. Bisio, A., Sciutti, A., Nori, F., Metta, G., Fadiga, L., Sandini, G., \& Pozzo, T. (2014). Motor contagion during human-human and human-robot interaction. PloS one, 9(8), e106172. 